\title{
MOMENTI ED ASPETTI DEL PARLAMENTARISMO MEDIEVALE
}

\author{
A. Marongiu
}

Come tanta altra parte della nostra vita associata, anche le istituzioni parlamentari, oggi parte rilevante dei nostri ordinamenti civili, sono, per quanto modificate ed adattate ai tempi, un'eredità tramandataci dal medioevo. Parliamo, ben inteso, di istituzioni parlamentari in generale, piuttosto che di forme particolari o determinate, come il parlamento inglese, il quale ha, tra le altre, il vanto di una continuità ininterrotta ormai da vari secoli, e badiamo - questo è essenziale - non al nome ma alla sostanza, cioc̀ senza fare distinzione tra' parlamenti propriamente detti e Cortes oppure Diete (Tag o Dag) dei paesi germanici. Parliamo, inoltre, di parlamenti nel senso, congeniale allo sviluppo storico del termine, di assemblec rappresentative e deliberative, senza cedere all'uso attuale di chiamarli assemblee legislative, tout court.

L'idea che ne abbiamo oggi, nel nostro presente, è quella asemblee, o collegi deliberanti, collocati al sommo e anche al centro, dei rispettivi Stati, davanti al sovrano, nei paesi monarchici, o al presidente della repubblica dall'altro, come espressione della volontà del popolo, e come ente autoritario e potestativo, impersonante la sovranità dello Stato. In origine, all'inizio della loro concreta presenza, i parlamenti, quelle assemblee alle quali abbiamo ripetutamente accennato, erano qualche cosa sia di meno autore- 
vole sia di meno consistente. Storici e scrittori dei secoli passati sono andati, o per mancanza di critica e di senso storico oppure nell'illusione di trovare agganci ed appoggi alle loro aspirazioni, ritrovando diretti precedenti di tali istituzioni nei comitia romani o nelle assemblee carolinge e simili. In tempi meno lontani si è andati cercando dei prototipi o dei precedenti, in assemblee locali (p.es. in Islanda o nell' isola di Man o nei primi cantoni svizzeri): ‘̀ chiaro però che, valessero quel che valessero, esse erano assemblee totalitarie le quali non avevano niente di simile alle istituzioni rappresentative, a livello di Stato territoriale o almeno provinciale, come quelle cui solo è dedicato il nostro studio.

Ovviamente, i parlamenti medievali non sono nati dal nulla, né dalla, più o meno geniale, invenzione di un sovrano particolarmente lungimirante. Essi hanno avuto come punto di partenza l'uso generalmente seguito dai sovrani -qua e là, come nella monarchia visigotica di Spagna, con i suoi concilios o in quella anglosassone con i Witenagemâts, anche prima dell' età feudale, ma più generalmente in quest'ultima - di convocare più o meno frequentemente a corte $i$ titolari, allora tanto vicini ai monarchi, delle maggiori dignità ecclesiastiche e laiche, i loro diretti vassalli, per assicurarsi la loro collaborazione e soddisfare alle loro frequenti querele ed istanze e per domandarne, appunto secondo il principio base del sistema feudale, consiglio ed aiuto.

Piuttosto che di assemblee, cioè di qualche cosa di coerente e di unitario, in senso associativo, si trattava di incontri, di discussioni tra il sovrano ed i singoli convenuti, individualmente presenti, ciascuno, per conto suo. Nel 1882, lo studioso francese Alphonse Callery, ponendosi il problema delle origini degli 'stati generali' del suo paese, e parlando delle assemblee della monarchia 'capetingia', sottolineava il carattere, consultivo e non deliberativo, di queste ultime. Constatava che, in esse, il sovrano chiedeva, ed otteneva, consiglio: egli informava e si informava ed i presenti rispondevano. Più di una volta, alla richiesta ed offerta di giustizia (il sovrano, nelle convocazioni, aveva detto che voleva proprio questo, di soddisfare le richiesta di giustizia, le querele o i gravamina dei suoi fedeli) corrispondeva la domanda - più o meno formale - o l'affettuosa preghiera di un aiuto o sussidio, in uomini o in denaro, talvolta soltando in moneta. Ma, chiamati come erano stati a titolo personale, ciascuno, obbligandosi, prometteva per sć, di sua volontà, per l'ammontare che personalmente lo con- 
cerneva, indipendentemente, almeno dal punto di vista giuridico, dagli altri, molti o pochi, disposti a soddisfare o non alle richieste dello stesso sovrano. Non c'era nessuna deliberazione collegiale (la quale avrebbe richiesto appunto l'esistenza di una collettività deliberante in comune, come unità) bensí soltanto una pluralità o moltitudine di individui. Il sorgere, il costituirsi, sia pure - come prima - in seguito a chiamata regia, di una entità associativa comprensiva e soprastante alle singole individualità sarebbe stato uno sviluppo successivo, costituente e caratterizzante le assemblee deliberative.

La differenza, in fatto, tra i due tipi di riunioni multiindividuali o di assemblee, non sfuggì, nel 1883-84, allo storico delle Cortes Manuel Colmeiro. Parlando di esse, infatti, egli constatava che parecchie di esse fossero, anziché delle Cortes nel senso moderno della parola, soltando dei verdaderos concilios al estilo wisigodo. Successivamente, nel 1895, S. Bové, nel suo volume sulle Institucions de Catalunya e più tardi, un eminente giurista, ed un autorevole storico del diritto, rispettivamente Guillermo de Brocà e Salvador Minguijón, distinguevano anch'essi, nella storia e prassi di quelle prime assemblee, le verdaderas Cortes o Cortes de derecho da quelle che tali non erano state. Nel mio volume del 1949 , su L'istituto parlamentare in Italia dalle originial 1500, studiando preliminarmente e, per quanto potei, approfonditamente l'argomento identificai, in quella che chiamai 'la monarchia temperata del l'epoca postcarolingia', due tipi di esse, uno generico e imperfetto, cui detti, o proposi, il nome di preparlamenti, e l'altro, di assemblee costituenti delle vere e proprie istituzioni, deliberative e rappresentative, il solo degno del nome di parlamenti (o, naturalmente, di vere e proprie Cortes) nel senso evolutivo ed evoluto del termine. Un passo, in qualche modo decisivo, per l'enucleazione di tali due momenti, o tipi, di grandi assemblee e per la fortuna del vocabolo 'preparlamento' fu compiuto -ritengo opportuno il ricordarlo- quando, nel 1954, l'ora de. funta illustre studiosa Miss Helen M. Cam ed io stesso fummo incaricati dalla Commissione internazionale per la storia delle assemblee di 'stati', di preparare una relazione, per il successivo X Congresso internazionale di Scienze Storiche (Roma, 1955), sullo stato degli studi, nel mondo, intorno alle assemblee medesime. Dovetti, in tale occasione, riprendere in esame, criticamente, tutta la questione. Persuadere un'inglese ad entrare nello spirito di 
un europeo del Continente e ad accettare una qualunque definizione concettuale, terminologica, era impresa ardua. Tuttavia mi riuscì. Approvata ed inserita nella già accennata Relazione, la proposta fu, quindi, presentata agli studiossi di tutto il mondo. Possiamo dire che abbia avuto partita vinta. Essa non è stata né contestata né ripudiata, neppure da coloro i quali, anziché di preparlamenti preferiscono parlare di assemblee prerappresentative ( $\boldsymbol{e}$ di assemblee rappresentative).

Al fondo, come disse agli inizi di questo secolo il grande gíuspubblicista Georg Jellinek, di ogni trasformazione istituzionale, c'è, sta, l'elemento psicologico: qui però, in questa evoluzione della quale abbiamo indicato soltanto il punto di partenza, soccorse e contribuì anche un progresso, un evento, potremmo dire tecnico (di diritto come norma tecnica parlò già un nostro storicofilosofo), vale a dire l'emergere del fenomeno, o del momento, associativo tanto in campo ecclesiastico quanto nella vita civile ed il venire alla luce, per merito, soprattutto, della dottrina canonistica (di Sinibaldo dei Fieschi, o papa Innocenzo IV, in particolare) del principio e dell'istituto della rappresentanza. Ad un certo punto, tanto la partecipazione delle comunità religiose, delle città autonome, delle grandi assemblee che abbiamo detto preparlamentari, concorre a far si che queste assumano ed acquistino, nel deliberare e per deliberare, anche coscienza di essere un tutto unitario ed aspetto e carattere rappresentativi: dal basso per le elezioni e i mandati ricevuti e dall'alto con l'esprimere voti e consensi obbligatori per tutti i membri delle rispettive maggiori comunità settoriali, persino, le maggiori assemblec, nei confronti di tutta la popolazione del paese soggetto al sovrano che le aveva convocate. Siffatta evoluzione non fu, naturalmente, da par tutto o egualmente semplice e rapida. $\overrightarrow{\mathrm{E}}$ molto più facile constatarne il punto di arrivo che le modalità, le fasi, e gli aspetti specifici.

$\hat{\mathrm{E}}$ oggi opinione comune, come si accennava, che la trasformazione della quale andiamo cercando non solo o non tanto gli effetti ma la genesi abbia avuto per sede quelle stesse, più o meno larghe, assemblee che i sovrani medievali erano uși convocare, per ogni dove, in occasione delle maggiori solennità religiose o di altre ricorrenze particolarmente sentite nei rispettivi paesi. La monarchia mancava, un pò da per tutto, di sede fissa. I sovrani - che non erano, si direbbe, mai soli- erano in continuo movimento; viaggiavano essi, con la loro corte e quindi la loro giustizia, della 
quale erano essi stessi organi, quindi la tenuta o convocazione di assemblee, i 'colloqui' o 'trattati' o 'curie', o 'parlamenti', o 'consigli' era cosa se non proprio di tutti i dodici mesi dell'anno (c'erano di questi nei quali il clima o altri fatci, quali le raccolte ecc., sconsigliavano di distrarre i sudditi dalle loro sedi) comunque frequente: le usanze feudali e i-diritti di regalìa (fodro, o 'albergaria' e simili) davano agli stessi monarchi il mezzo di viaggiare e di mantenersi a contatto (e con l'ospitalità!) di chiese, conventi (le feste religiose servivano anche per questo!), principi ed altri grandi signori feudali. Al sovrano facevano corona, con o senza quest'ultima parola, che troviamo, per esempio, in uso in due pacsi lontani l'uno dall'altro, la Sardegna e l'Ungheria, gli alti ufficiali, ecclesiastici e laici, della sua corte, normalmente coloro che convivevano con lui e ne erano i compagni anche nei suoi spostamenti, nei banchetti, nelle cacce ecc.e i tanti altri dei territori in cui si recavano o che venivano chiamati, appunto, a partecipare ai surriferiti incontri o riunioni fuori sédo del Consiglio regio, comunque denominati. La giustizia, la pace interna, le tregue e gli accordi all'interno del paese non crano sempre osservati e rispertati ed i sovrani non erano sempre giusti o imparziali nei loro favori, e grazie, e le riunioni in questione non erano sempre così casuali o prive di significato, semplicemente informative, ma erano state, anzi, talvolta provocate da clamores, o querele o querimonie di questo o quel vasallo, affinché essi rendessero loro giustizia. Il XIII secolo vide tale richiesta di giustizia, e di assemblec, assumere, in Inghilterra ed in Aragona, importanza ed aspetti addirittura rivoluzionari, in assemblee acefale che osayano intimare ai sovrani di comparire davanti a loro.

Fra tali e tanti nomi (ma non è detto che ce no fosse sempre uno) di riunioni allargate del Consiglio regio, e specie da quando questi incontri finirono per diventare un fatto giuridico deliberativo e rappresentativo, fini per prevalere, in Italia e in Inghilterra, la parola parlamento (in latino parlamentum, ma Oltremanica mutato in parliamentum), parola, almeno per lungo tempo, priva di un significato tecnico-giuridico, tanto che, ancora nella metà del su ricordato secolo, il lessicologo Giovanni da Genova, nella sua Summa quae vocatur Catholicon, lo citerà come un termine del linguaggio popolare (colloquium, consilium vel concio, quod vulgo dicitur parlamentum). La parola, dunque, sembrava voler dire, soltánto, discussione, contradittorio, diálogo, a più voci. Era 
anch 'esso un vocabolo nuovo, un neologismo. La sua apparizione non risale (almeno nelle testimonianze erudite) più indietro della celebre Chanson de Roland, probabilmente della fine dell'XI o del principio del XII secolo, sono stati oggetto di studi validi ed interessanti, anche se, forse, meritevoli di integrazioni c rilievi. Meno studiata è stata, invece, forse la sorte, la semantica ricca e complessa, della parola latina curia e del suo equivalente corte, o cort, la qual ha avuto in quel medesimo periodo altrettanta importanza e, certo, non minore diffusione in molti paesi, anche all' infuori dei territori iberici. La fortuna delle parole ha qualche cosa di misterioso. Una stessa parola può indicare, in tempi successivi, diverse realtà; altre volte, al contrario, la stessa realtà, continuando, può essere indicata o segnata in modi e con termini differenti.

Un autorevole storico francese della prima metà di questo secolo, Charles Petit-Dutaillis, ha scritto, alludendo alla parola parlamento ed al suo significato finale, soprattutto riguardo all'Inghilterra, che les institutions humaines $n$ 'existent réellement pas que quand leur a donné un nom. Tale nominalismo, tale criterio lessicale, è però tutt'altro che persuasivo: esso mette in ombra, sottovaluta, la sostanza vera e concreta della realtà. Il rilievo, per esempio, di uno scrittore inglese, J.R. Seeley, secondo il quale 'in Inghilterra sembra più facile modificare lo stato delle cose anziché il loro nome'ha, ci sembra, valore generale per tutto ciò che si riferisce al mondo medievale. Ritornando indietro alla parola curia, o corte, ed alla sua lunga storia, d'altronde, vediamo chiaro, ancóra prima di registrarne la presenza e la vitalità nei paesi di civiltà iberica, che essa abbia indicato tanto assemblee limitate, preparlamentari quanto istituzioni rappresentative piene ed orgogliose della loro forza. Avendo studiato, in altra sede, la storia della parola 'stato', ci rifiuteremmo di ammettere che lo Stato, o gli Stati, non esistessero anche quando la corrispondente realtà era detta res publica o cosa pubblica, regno, città o altro.

Le parole sono, dunque, una cosa, più o meno importante, che perô non basta ad imbrigliare, spesso, la realtà. Il parlamento come istituzione, abbiamo detto, politica rappresentativa e deliberativa è cosa assai più tarda del termine. L'esempio inglese sembra molto probante, se, però considerato senza prevenzioni, senza, cioè, accedere al mito, puramente agiografico, di un 'parlamento modello' del 1295 e cosí del genere. Parlamento era, e lungamente fu anche in Inghilterra, non il nome di una nuova istituzione, 
ma soltando quello dato al riunioni apposite, pubbliche e solenni, del Consiglio del Re, tenute alla presenza, oltre che del sovrano, dei suoi maggiori vassalli ecclesiastici e laici: vale a dire una particolari riunioni di esso dedicata soprattutto all 'esame e alla discussione, ed alla riparazione, dei torti che gli invitati alla riunioni o altri ricorrenti ritenevano di aver subito; in questo senso ed a tal fine, un documento di critica e di opposizione alla politica regia, le Provisions di Oxford del 1258, domandavano, o piuttosto imponevano, ad Enrico III, treis parlemenz... par an.

Secondo l'anònimo opuscolo Fleta, da datare, probabilmente, intorno al 1290, nei suoi parlamenti il Re siedeva circondato dai membri del suo Consiglio, teneva la sua corte...vi si risolvevano i dubbi sorti nel corso dei giudizi, venivano disposti i provvedimenti necessari alla riparazione dei torti, si dispensava giustizia come ognuno meritava. Le già citate Provisions, andando un poco più in là, avevano detto che essi venivano indetti per trattare de l'estat $d u$ reaume...et des communes besoignes du roy et du reaume. 1 Rotuli parliamentorum mettono in continua evidenza le 'petizioni' o i bills, ossia le richiesta di giustizia ora di questo ora di quel vassallo, o città, e persino di una, difficile a identificare, 'Comunità del regno'. Tuttavia sarebbe azzardato dire che essi fossero qualche cosa di ben diverso dai tanti 'Consigli' o 'Concitia', talvolta qualificati 'grandi', del regno, che i sovrani non avevano smesso di riunire, per fini vari e diversi, in quello stesso periodo. Di molte riunioni manca ogni denominazione, mentre, con frequenza e decisione, anche le assemblee riunite 'a parlamento' esaminavano questioni anche d'indole politica o finanziaria. Un celebre passo dello Statuto di York del 1322 stabiliva che tutto ciò che poteva influire sullo stato del re, del regno o del popolo doveva essere trattato e stabilito, appunto 'in parlamento', dal re, con l'assenso dei prelati, conti e baroni e della comunità del regno: 'cosi come era stato fatto in passato'.

Con caratteristiche più o meno analoghe, i sovrani dei vari regni iberici tenevano le loro 'curie', o corti, ora più ora meno 'piene'. Il termine curia, d'altronde, non poteva non far pensare ad una riunione giudiziaria, col re sedente pro tribunali, con la citazione a comparirvi di tutti coloro i quali dovevano intervenirvi, con la dichiarazione di contumacia degli assenti non giustificati o non rappresentati, con la presentazione dei mandati ai procuratori e per presentazione dei gravami ecc. Da queste riunioni, o as- 
semblee in cui ciascuno sedeva, intorno al sovrano, nel luogo fissato dalla consuetudine in relazione alla sua appartenenza ai vari gruppi e categorie, -alle quali i sovrani facevano le loro 'proposizioni' promettenti e offrenti giustizia e chiedenti, viceversa, consiglio ed aiuto- nacquero, in tempi o in occasioni diversi, - ma con mutamenti formali poco percettibili- quegli stessi organi collegiali rappresentativi e deliberativi che poi fecero tanto cammino e che abbiamo deciso di chiamare 'parlamenti', o 'corti', nel senso pieno e proprio della parola.

Qualcuno - e precisamente Jan Dhondt - ha detto che l'origine delle istituzioni rappresentative sia un problema addirittura immenso e che per farvi un po' di luce occorra, soprattutto, ricercarne il perché e il come. Da parte nostra, restiamo fedeli sia alla concenzione che nulla nasce dal nulla sia ad alcune ipotesi, in linea alternativa, formulate in un nostro volume del 1949. Le seguenti:

a) i convenuti a!le grandi assemblee, convocate dal sovrano, oppure, irregolarmente, da qualche altro grande signore o da più signori uniti in lega, rendendosi conto di essere non più o non tanto un piccolo gruppo di persone, ma di esprimere interessi ed intenti della collettività dei sudditi, si trasformano appunto in corpo o collegio unitario e collettivo, come persona o soggetto nuovo e diverso, e con un nuovo ed altrettanto unitario potere decisionale; b) il re, o principe territoriale, o per ragioni di tornaconto o di opportunità, cioè persando soprattutto al suo proprio interesse, o per un'intesa in tal senso con gli stessi convenuti all'assemblea, investe questa medesima (al fine di ottenere, con la sua collaborazione, delle imposte o degli aiuti e sussidi volontari: non dimentichiamo che, secondo la concezione medievale, questi sono soltanto dei doni liberamente consentiti) della funzione di rappresentanti della intera collettività: trasformando così, egli stesso, la pluralità dei convenuti in unità operativa e deliberativa; $\mathrm{e}$ dando, così, vita ad una nuova istituzione; c) le forze politico-sociali, prevalenti nel paese, riunite in leghe ed organi assembleari, persuadono, o costringono, il sovrano restio ad accoglierne le querele e i gravami a licenziare questo o quel suo consigliere o ministro, a tornar sopra le sue più invise decisioni e a fornire garanzie (come più volte avvenne in Inghilterra $\mathrm{e}$ in Aragona) contro il ripetersi degli abusi già lamentati. Il re legittima, pro bono pacis, tali comportamenti ed accetta, implicitamente o esplicitamente, l'impeg- 
no a non assumere decisioni d'importanza vitale per tutti senza il consiglio (per non dire anche il consenso) dei partecipanti alle grandi assemblee; d) l'instaurazione di questo inizio o riconoscimento della esistenza di un corpo o collegio rappresentativo può essere determinato anche da simmetria istituzionale tra i vari domini di uno stesso sovrano o per introduzione o trapianto in, o tra, differenti territori per effetto del loro entrare a far parte, appunto, dei domìni di uno stesso monarca.

Parlando del costituirsi delle assemblee rappresentative (Standen, états) nelle piccole formazioni geopolitiche dei Paesi Bassi, l'anzidetto J. Dhondt ha anche parlato di una reazione dei sudditi nei confronti dei loro principi, che scatta presso a poco immancabilmente col verificarsi di circostanze favorevoli ed è, invece, frenata nel suo sviluppo quando la situazione stessa si modifica. Che questo avvenisse o potesse avvenire è cosa semplice e chiara: il cammino della libertà è sempre irto di difficoltà e di ostacoli.

Particolare tipico dei parlamenti medievali è - fuorché nei momenti rivoluzionari- l'immediatezza dei rapporti tra le assemblee e i sovrani. Gli Inglesi - ma non essi soltanto- dicono che il re è capo e fondamento del parlamento. Dovunque, entrano e fanno parte delle assemblee soltanto coloro che il sovrano ha convocato personalmente of fatto scegliere (in Inghilterra dagli sceriffi delle contee e altrove dalle amministrazioni locali o, eventualmente, da altri organi). In linea di principio: $\mathrm{I}^{\circ}$ ) il sovrano convocava l'assemblea dove e quando lo rinenesse opportuno, con un congruo termine di anticipo; $2^{\circ}$ ) le convocazioni, anche se talvolta graduate nell'espressione del comando, per riguardo ai prelati, rivestivano l'aspetto di una citazione e l'inadempimebto a tale ordine costituiva, si diceva, contumacia, sanabile però con la, sia pure ritardata, comparizione. Poiché tuttavia, si scoptì, ad un certo punto, che partecipare alle assemblee era cosa che portava con sé dei vantaggi d'indole materiale, di prestigio o d'altro, fu visto, e non è sfuggito agli studiosi, che l'ufficio di deputato, o di procuratore, nuncio e simili, venisse ambito c diventasse persino, qua e là, appannaggio di determinate famiglie. Soprattutto importante, ed indiscusso, era il potere del sovrano di convocare l'assemblea quando lo avesse voluto (tale regola non poteva venir meno che per sua stessa autolimitazione) a suo prudente arbitrio; di tenerla in vita finché egli avesse voluto; di presiederla o di farla presiedere da un suo delegato; di prorogarla o di 'licenziarla' o, come 
diremmo noi oggi, di scioglierla, autorizzando i convocati a fare ritorno alle loro rispettive sedi. Gli stessi ordini parlamentari, le assemblee, cercarono a più riprese d'interferire limitando tali prerogative, ma con risultati sempre effimeri $e$, ben inteso, parziali. Parimenti, il sovrano restava libero di concedere o meno la sua sanzione, cioè di rendere esecutivi, i voti parlamentari bills, capitoli o atti di Corte o altro che si chiamassero. Principio tuttavia molto autorevole (anche se non certo al cento per cento, perché i sovrani si limitavano ad osservarlo, per lo più, ma non ad ammetterlo formalmente, esplicitamente), era che le disposizioni di legge votate in parlamento non potessero, una volta sanzionate dal re, essere annullate o mutate se non in forza di nuovi voti parlamentari. Certe volte, d'altronde, le assemblee, invece di limitarsi a domandare la semplice sanzione regia, dei loro voti, ottenevano che il re li sancisse a titolo di contratto, pro praetio, e ciò creava nuove difficoltà, gravi limiti al potere legislativo del sovrano.

A questo punto, sopravvengono delle considerazioni non più o non tanto d'indole giuridica ma storica. Il sovrano poteva, certo, non accogliere le domande, $\mathrm{i}$ voti, le proposte parlamentari (in realtà le forme erano sempre rispettate, e piuttosto che dire senz'altro di no, egli diceva che ci avrebbe pensato o usava un'altra espressione meno impegnativa: i parlamentari parlavano sempre, molto rispettosamente, sotto forma di supplica ed il sovrano non mancava mai di manifestare loro la sua benevolenza, il suo affetto); ma, dal canto suo, il parlamento poteva condurre in lungo, per un migliore e più adeguato esame, l'accoglimento della, amorevole ed affettuosa, richiesta sovrana di aiuti, sussidi o sovvenzioni: e persino, con procedimenti più o meno ostruzionistici, negarglieli del tutto; o sottoporre la concessione a condizioni e patti più o meno onerosi. Normalmente, dunque, il voto dei sussidi era condizionato - con chiara pratica del do ut des - appunto con o all'esaudimento delle domande parlamentari, all'accoglimento dei gravami ecc.

A differenza dalle assemblee preparlamentari, nelle quali gli impegni erano assunti dai singoli, i parlamenti, cortes, diete, ecc., concedono auti o sussidi obbligatori per tutti i sudditi, quindi anche per gli assenti e i non convocati. Con cura puntuale ed inalterabile, dicono, ripetono, e pretendono che ciò sia detto ed am. messo anche dal sovrano, nel concedere gli 'aiuti', che lo fanno a titolo grazioso, per pura liberalità, senza esservi tenuti, una volta 
tanto, senza che tale eccezione debba o possa aver seguito o costituire precedente. Spesso, le stesse assemblee intervengono nel regolarne, direttamente o (come, per esempio, in Catalogna per mezzo di altro apposito organo) e la riscossione e l'impiego al fine voluto (c.d. appropriation, in inglese), pet evitare, cioè, che i mezzi apprestatj per un certo fine vengano deviati o stornati per scopi diversi.

Il sovrano e l'assemblea parlamentare realizzano, continuano e rinnovano di volta in volta il dialogo, il colloquio, tra il Re e il suo Regno. Eccezionalmente, in caso di crisi dinastiche o di minorità del sovrano, le assemblee partecipano alla scelta del successore oppure del consiglio di reggenza. Il parlamento rappresenta il paese, riceve ed invia, talvolta, degli ambasciatori e ratifica o garantisce trattati internazionali. Che esso rappresenti tutti $i$ sudditi è però soltanto una finzione giuridica. Si tratta di una rappresentanza che prescinde, largamente, dalle volontà dei rappresentati. Ciò è evidente sia per quanto riguarda i signori feudali, ecclesiastici e laici considerati come rappresentanti degli abitanti dei loro rispettivi territori, sia per quanto riguarda il gruppo dei rappresentanti cittadini (o, in Inghilterra, delle contee e dei borghi: di pochi borghi soltanto e senza alcun rapporto con la qualità e la popolazione di questi!), considerati come espressione di tutto il paese libero dalle signorie feudali. È una finzione, si è detto, ma comunemente ammessa.

Altro momento tipico è il mandato. Esclusi $i$ pochi alti prelati (quelli che in Inghilterra verranno chiamati i Lords spirituali) e i magnati e gli altri nobili ad essi assimilati, i quali sono convocati personalmente e finiscono per credere di aver diritto alle convocazioni (anch'essi perồ, se impediti a comparire, devono farsi sostituire da un loro mandatario), tutti gli altri (comunità ecclesiastiche, città e centri minori, come 'ville', i botghi e le contee), nonché, dove abbiano vita (p.es.in Piemonte e in Friuli) le consorterie nobiliari sono tenuti ad inviare alle assemblee dei procuratori o sindaci, muniti di mandato 'pieno e sufficiente'. Una delle prime cose da fare, all'inizio delle sessioni parlamentari, è, proprio, la verifica dei titoli a comparire dei chiamati intervenuti al parlamento. Doveva essere, era, in origine un compito riservato ai funzionari regi: col tempo vi participano gli stessi ordini parlamentari. Sarà 'verificata', da parte del parlamento, la stessa delega sovrana al rappresentante da lui inviato, per sostituirlo, in assemblea. 
Non c'è nessuno il quale ignori che i deputati del 'terzo stato', agli 'stati generali' francesi venissero, dai loro elettori, muniti di un mandato 'imperativo'; non si trattava però di una regola seguita in altri parlamenti se non per eccezione. In generale, altrove, i mandati attestavano piena fiducia negli eletti con promessa di fermo e rato di ciò che essi avessero fatto nell'esecuzione di essi, senza limiti né predeterminazioni contenutistiche. Qui per alium facit, per se ipsum facere videtur, punto e basta. Era, tuttavia, prassi frequente la consegna al mandatario procuratore, di dati, istanze, gravami da presentare e di cui chiedere soddisfazione. Ciò vincolava, evidentemente $\mathrm{i}$ deputati o procuratori, a determinati adempimenti, ma non voleva dire che essi non fossero liberi di svolgere la loro funzione liberamente, a loro prudente arbitrio. La cosa sarebbe stata, naturalmente diversa, era diversa, nel caso che ad essi procuratori fosse stato conferito soltanto un mandato ad audiendum et referendum. Di fatto, si verificò anche questo, ma non fu mai una regola. I sovrani vi erano nettamente contrari, in quanto ciò non avrebbe potuto non ritardare o rendere difficile una qualche decisione. Le Corts catalane del 1419-20 proposero esse medesime al re Alfonso V un 'capitolo di corte' vietante proprio la partecipazione alle assemblee di chiunque avesse assunto in alcun modo l'impegno a votare in un modo o nell'altro: volevano, infatti, che ciascúno accedesse all'assemblee con 'libero arbitrio'. Per completezza, dobbiamo per altro aggiungere che a Barcellona una 'commissione di ventiquattro' (vintiquatrena de cort) ed a Cagliari una di tredici membri (tretzena de cort) assisteva i rappresentanti parlamentari delle due, consigliandoli e guidandoli.

Con divisione più o meno rigida e completa - $\mathrm{e}$ ad eccezione delle riunioni plenarie o solenni delle dichiarazioni o 'proposte' regie e delle risposte collettive - i vari parlamenti, Cortes e Diete funzionavano e deliberavano non già unitariamente ma divisi in case, o camere, o stati o bracci, ordini e simili. Non si trattava però soltanto di modalità operative ma di strutture ed organizzazione. Gran parte delle istituzioni parlamentari medievali, quasi tutte, erano, nella loro concreta sostanza, delle assemblec di 'stati', cioc̀ fatte, composte, di parti o collegi minori, detti, appunto 'stati', 'ordini' ecc. In Inghilterra, dove esistevano due camere (parti della stessa 'casa' del parlamento) la cosa era meno visibile o forse dubbia perché ciascuna delle due Camere era composta di elementi eterogenei (lords spirituali e lords temporali nella 'Camera 
alta', deputati delle città o borghi e dei cavalieri delle contee in quella 'bassa'): altrove, per lo più, il corpo parlamentare era composto di tre 'stati', o 'bracci', grosso modo corrispondenti, ma solo dal punto di vista giuridico non da quello, molto più articolato, sociale, alla divisione della popolazione in classi: ecclesiastici, nobiltà, 'terzo stato'. Per qualche tempo, a quattro 'stati', o bracci, ci sono soltanto le Cortes aragonesi, per il distacco dei richi homines, magnati, baroni dai semplici 'cavalieri'. Più tardi, il fenomeno si ripete, in vari paesi del centro Europa, per la costituzione deIlo 'stato' dei contadini.

Quando o come o perché tale divisione si sia venuta alla luce è cosa estremamente difficile a dire, e persino a supporre. Lo storico francese E. Perroy ha supposto che la ripartizione, o associazione, dei partecipanti alle grandi assemblee in categorie o ordini sia stata un'antichissima abitudine, risalente, addirittura, alle grandi riunioni di palazzo dell' epoca carolingia: senonché, lo stesso autore opinava che essa non dovette avere avuto allora, e neppure agli inizi del XIV secolo, il significato che ciò avrebbe avuto in seguito, di divisione fondamentale in clero, nobiltà e terzo stato, costituenti, ciascuno, una classe o parte dell'assemblea. Mancava, in origine, infatti una differenza netta e profonda fra tutti, convocati come erano a titolo di vassalli regi. Forse - ma qui azzardiamo un'ipotesi oltremodo difficile a verificare - momento critico, o catalizzatore, tanto di tale... statificazione quanto di tale triplicazione dovette essere quello dell'ammissione, o introduzione, in blocco, delle rappresentanze cittadine (fino ad allora tale presenza era stata soltanto eccezionale, isolata e sporadica, limitata ad una o due città), elemento, evidentemente, non assimilabile né al clero né al baronaggio.

Forse prima che altrove, la strutturazione delle assemblee rappressentative in 'stati', tale novità giuridico-semantica, si è avuta in Francia, nel 1316, quando Carlo di Valois, in procinto di partire per la Crociata, ringraziava dell'aiuto concessogli gli estaz (prelati, nobili e comuni) 'della lingua di Normandia' (langue d'oil??). Sempre in Francia - purtroppo la documentazione è scarsa e frammentaria - la ritroviamo ormai pienamente in vigore negli anni Cinquanta dello stesso secolo. Per esempio, l'art. 7 di una Ordonnance del 22 dicembre 1355 affermava che il Reggente del regno aveva agito col consenso dei trois estaz; l'art. 27 di essa precisava, inoltre, che l'aide che questi gli avevano concesso poneva 
termine ad ogni altra imposizione, ma che, in caso di ripresa delle ostilità, i embri des trois Estaz avrebbero riesaminato il da fare. Altre Ordinanze, del 1356, prevedevano riunioni separate dei tre stati' dei paesi di lingua $d$ 'oül e di lingua $d$ 'oc: i membri di tali assemblee esprimevano i loro pareri in seno al rispettivo 'stato'; venivano scelti dei 'deputati' di 'ciascuno stato', e così via, per tutti tre gli 'stati'.

Quanto alla Spagna, forse, il fenomeno si verificò, prima che altrove, in Navarra, regno ai due lati dei Pirenei, gravitante per lungo tempo verso la Francia. Nel settembre del 1319, stando a Parigi, il re Filippo di Valois il Lungo (alla spagnola el Largo) pres. tava davanti ai procuratores et nuncii degli estados e pueblos il suo giuramento sottoscrivendo, in tale occasione, vari atti e latini e spagnoli in cui i tres estados e gli status del regno erano ripetutamente menzionati. Un altro documento ufficiale, dello stesso reg. no pirenaico, del 1329, conteneva ben sei volta la parola 'stati', o 'tre stati'. E in Catalogna? Le gravi lacune della documentazione non consentono alcuna certezza per il periodo precedente al 1352 , anno nel quale invece troviamo, nel corso delle Corts tenute prima a Perpignano e poi a Lérida, già in atto i tre Braces (al singolare o braf o bras) ossia i i tre Brachii e, nominati da essi, dei Clavarii, ciascuno per son Bras. Nulla di preciso saremmo in grado di dire (ce ne rincresce, ma ci auguriamo che altri studiosi ci illuminino al riguardo) intorno alle prime apparizioni dei 'bracci', o 'stamenti', nelle altre analoghe Cortes, tanto meno sullo sdoppjamento, in Aragona, del 'braccio militare'. Quanto, tuttavia, al regno di Valenza, vorremmo ricordare due cose: $I^{\circ}$ ) l'accenno ai bracci o stamenti manca negli atti delle Corts del 1349, nei quali si parla dei 'prelats, richs homens, cavalletos e generoses e prohomens de les ciutats e viles del dit regne' ed il re Pietro IV dettava seis rubricas 'ab acord consell é expres consentiment de tots los dessus dits é à humil suplicaciò de aquells'; $2^{\circ}$ ) esso è invece presente nelle Corts del 1364, pure tenute a Valenza, nella quale lo stesso sovrano riceveva da 'omnes qui in dicta curia erant, tam de brachio ecclesiastico, quam militari, quam etiam civitatum et villarum regalium', a titolo di subvencio e di donum, la somma che egli stesso aveva richiesto per la difesa del regno: dei tria brachia medesimi si parlò anche, p. es., nella sessione del 1369-70. L'equivalente inglese estat, più tardi state, o estate, non fece, probabilmente per le ragioni già ricordate, che poche e tarde appari- 
zioni: forse per la prima volta nel 1382, in un voto parlamentare, il quale parlava appunto di estates, poi nel 1399 - quando i Rolls, o Rotuli, del parlamento usarono l'espressione 'davanti al Re e a tutti gli stati nel presente parlamento'- poi ancora nel 1421 in cui si parlò dei tres status regni'; di due 'camere' si parlò, sembra, soltanto intorno al 1450 .

Che cosa i parlamenti fossero in realtà è cosa che da gran tempo si è cercato di precisare, e di definire: forse la più antica definizione è quella che ne dava, scrivendo, sembra, nel 1438-39, il valenzano Pere Belluga. Curia (cioè Cort) era la congregatio populi facta in certo loco communi a principe vel ab alio habente potestatem Curiam celebrandi, ad quam debent solemniter vocari... Archiepiscopi, Episcopi, Duces et caeteri Magnates, nobiles, milites et generosi et regni proceres, convocati de iure pro reparatione status pacifici regni et illius utilitatis et ut singulorum status iugiter conservetur illaesus pro iustitia et pace componenda ac ad regem honorem augendum. In realtà $i$ sovrani non cercavano nelle assemblee onore e belle parole, bensì consigli e soprattutto auti, denaro. Tale definizione era a ben vedere un pò troppo aulica e parziale. Il resto delle parole del giurista valenzano era, invece, preciso e probante. La curia è convocata, egli aggiungeva, con lettere monitorie o, più propriamente, di citazione, e chi non vi ottemperi è da dichiarare contumace (dopo i termini di grazia abitualmente concessi) a meno che, impedito, non si sia fatto personalmente rappresentare da un procuratore del suo stesso 'stato', munito di mandato ampio, illimitato.

Altre definizioni, nel secondo Settecento o nel primo Ottocento, relative agli Stände germanici, misero in evidenza più che altro il fatto che essi costituissero dei corpi privilegiati di sudditi. Sul finire, poi, dello stesso secolo precedente al nostro, nel 1888, il francese Léon Cadier, parlando degli 'stati' del Béarn, ne dette una definizione la quale riscosse molto successo. On entend, egli disse, par états provinciaux (ma le sue parole sembrano adattarsi anche agli 'stati generali') la réunion des trois ordres d'une province en assemblée regulièrement constituée, périodiquement convoquée, et possédant certaines attributions politiques et administratives dont la principale est le vote de l'impôt. Tale definizione presenta, come si è già accennato, vari punti deboli, come l'esigenza (mai completamente soddisfatta, anzi in contrasto col principio della discrezionalità delle convocazioni, da parte del 
sovrano) della periodicità e quella della regolare costituzione, la quale non è, certamente, cosa da poco: ma, pur con l'accenno alla competenza a deliberare in materia d'imposte, resta abbastanza nel generico. Come taluno ebbe già ad osservare, essa non corrisponderebbe se non a istituzioni completamente sviluppate e consolidate e non al periodo iniziale ed evolutivo del loro affermarsi. Tuttavia l'elemento tempo, il diretto o anche soltanto indiretto, collegamento tra i vari momenti, cioè le varie successive convocazioni (noi parliamo oggi, usualmente, di sessioni, ma allora ognuna di esse appariva un fatto a sé, indicato dal luogo e dalla data, si parlava, quindi, di tanti parlamenti quante ne erano state le riunioni), e l'ordinarietà oppure soltanto la relativa ordinarietà, o regolarità, di tali assemblee non possono non essere considerate come elementi di giudizio e manifestazioni o prove dell'esistenza di simili entità e del ruolo da esse tenuto nell'ordinamento e nella vita dello Stato.

Ma tale definizione peccava di genericità, nel parlare di tre 'stati' o 'ordini' senza però precisarne la natura di frazioni operative di un unico complesso e nel ridurre, implicitamente, la ragione dell'esistenza di tale corpo o complesso deliberativo e rappresentativo al voto dei tributi. La tripartizione infatti operava in seno ad un organo complessivo, esprimente una sua propria decisione collettiva, costituita dall'incontro di volontà dei suoi medesimi elementi costitutivi. In pratica, le deliberazioni erano prese non già nelle adunanze solenni e plenarie - tipicamente, di apertura e di chiusura delle sessioni- ma separatim, 'stato' per 'stato' o 'braccio per braccio', secondo le regole determinate da regole scritte oppure dagli usi, dal diritto cosidetto 'comune' ma, per meglio dire, comparato, ecc. Naturalmente, nessuna questione se i membri dei singoli 'stati' fossero stati unanimi (ma la formula nemine discrepante nascondeva talvolta qualche opposizione): diversamente, veniva fuori la questione se per maggioranza dovesse intendersi soltanto il maggior numero dei votanti, oppure la, causa di discussione e di contrasto nella pratica, maior et sanior pars, cioè il criterio che vota sunt ponderanda, et non numeranda. Sempre restando nel tema della deliberatività degli organismi parlamentari, non può essere dimenticato il diverso peso - nelle Cortes dei paesi della Corona d'Aragona- di un semplice voto contrario, minoritario e basta, rispetto al 'dissentimento'. Più o meno come, in Polonia, il liberum veto esso consisteva nell'oppo- 
sizione alla prosecuzione dei lavori parlamentari, e quindi a qualunque decisione, fino a quando i motivi, appunto, di tale veto non fossero stati esaminati e risolti nel modo prescritto per i gravami 'di corte'.

La già riferita definizione, del Cadier, lascia dunque completamente in ombra il carattere rappresentativo delle assemblee, che, invece, ne costituiva la funzione specifica e tipica, tanto nel riferire e chiedere al sovrano quanto nel dare o concedere a quest'ultimo, con decisione obbligatoria per tutti gli appartenenti alla collettività. Ciò non ha, in verità, bisogno di dimostrazione; ne presenteremo, tuttavia, qualche indicazione. Thomas Smith, scrivendo (De republica Anglorum) nella seconda metà del Cinquecento e parlando, precisamente, del parlamento inglese diceva che esso rappresentava e costituiva il capo, la testa, del popolo: ogni inglese vi si aveva per presente, di persona o per procura, quale che fosse la sua condizione, preminenza, stato, dignità, dal sovrano alla persona più umile del paese, ed il consenso del parlamento aveva il valore del consenso di tutti (everie mans consent). Ma non faceva che ripetere un concetto diffuso e comune. Infatti, nel 1480 , parlando dei 'Comuni', era stato detto che tutti vi erano rappresentati e ne facevano, quindi, parte. Sempre a proposito del parlamento inglese, l'autorevole $\mathrm{Ch}$. Mc Ilwain ha riferito che già nel 1365 esso venisse considerato come l'inticro 'corpo del regno'. Quanto alle Cortes iberiche, l'equivalenza delle loro riunioni a 'todos de la tierra', o a tutti i regnicoli era cosa non dubbia. 11 giurista Molino considerava il voto e consenso dei quattro 'bracci' delle Cortes d'Aragona come consenso degli Aragonesi. Il 19 dicembre 1410 il Parlamento di Barcellona e il 25 agosto 1422 le Corts dello stesso Principato dicevano di essere i rappresentati di tutti i Catalani. Lo stesso facevano i bracci parlamentari sardi nel 1495 , qualificandosi i tria brachia sive stamenta eius regni, ipsum regnum representantes. Altrettante testinianze potrebbero essere facilmente addotte, p. es. relativamente al 'Colloquio' della Patria del Friuli, all 'Ungheria ecc.

Una definizione, non solo più recente ma più attendibile, è, a tacere di altre, quella proposta dal prof. Émile Lousse, di Lovanio, come di'assemblea politica composta dei rappresentanti del ordine o degli ordini politicamente privilegiati di un paese, al fine (intendiamo così il vocabolo agissant, del testo francese) di vegliare, da un lato al mantenimento dei privilegi degli ordini, dei corpi e 
degli individui, così come alla conservazione dei diritti fondamentali del paese, e, dall'altro, per rendere al principe il corrispettivo dei diritti da lui riconosciuti e dei privilegi da lui concessi'. Essa concerne, tuttavia, più, o soltanto, l'aspetto politico istituzionale, il fatto piuttosto che il diritto. La formula in questione lascia peraltro in ombra l'intimo nesso tra le assemblee e l'ordinamento dello Stato, la loro qualità, appunto, di parte ed elemento della stessa compagine statuale, di istituzioni giuridiche, di diritto pubblico.

Qua e là, nel corso di questi brevi appunti, si è fatto richiamo ai giuristi del tempo ed alle loro opere di diritto parlamentare. Vengono sopratutto alla mente tre nomi: quelli del geronese Tomas Mieres, del 'cavaliere' e giudice dei gravami (provisor de greuges) alle Corts di San Cugat e avocato fiscale Jaume Callis e del già citato Pere Belluga. La loro dottrina esalta il ruolo delle Cortes, come rappresentanti dei rispettivi regni o principati di fronte al sovrano. Le loro opere hanno dei titoli molto precisi e significativi, dall' Apparatus, del primo super constitutiones curiarum generalium Cathaloniae, del secondo, soprattutto, l'Extravagatorium curiale e del terzo quello Speculum principum, che aveva il lungo e pretensioso sottotitolo In quo universa Imperatorum, Regum, Principum, Rerumpublicarum ac Civitatum subdito. rumque...iura ac officia, dignitates, mores, praesertim Regni Aragoniae varie ac dilucide pertractantur... e poi ancóra ex iure canonico, civili, constitutionibus regum Hispaniae, Jurisconsultorum, Historicorum ...variarum curiarum decisionibus, praeiudiciis ac votis controversiisque... Di essi, possono essere qui ricordati, per esempio, il brano del Callis sui diversi significati della parola curia, con particolare riguardo a quella catalana, prout in ea convocata residet dominus Rex, cum brachiis ecclesiae, baronum, militum et universitatum, civitatum et villarum Cathaloniae regiarum tractans de reformatione principatus Cathaloniae, e, del Be. lluga, tra l'altro, l'insistenza nell'affermazione del particolare valore delle leggi sanzionate dai sovrani pro praetio, leyes paccionadas, veri e propri contratti bilaterali, quindi non revocabili se non per mutuo nuovo e diverso consenso del Re e delle Cortes.

Le loro opere, fonte per noi di utili cognizioni, costituirono, in certo modo, per i Catalani e gli Aragonesi dei veri e propri testi di legge, poco meno che i testi veri e propri degli usi e consuetudini e delle vere e proprie leggi di cui davano notizia, non solo, ma fa- 
cevano testimonianza. Scrivendo, non sono passati tanti anni, ma in un regime ed un ambiente non, certo, ben disposti verso le prerogative parlamentari, un illustre storico catalano prematuramente scomparso e che tutti noi abbiamo ammirato, Jaume Vicens Vi. ves, accomunava tali scrittori, ed anche qualche altro minore, sotto il, per lui tutt'altro che elogiativo, epiteto di 'pattisti', cioè di sostenitori di un regime di patteggiamenti ed accordi tra i sovrani e le assemblee rappresentative. Chi scrive li ha difesi da tale giudizio sfavorevole, assumendo, o ricordando, che di accordi e contratti del genere era fatta un po' tutta la vita pubblica medievale, non ancóra piegata all'assolutismo monarchico, e che contrattualisti, o pattisti, furono anche vari secoli dopo in Francia dei sovrani tanto autoritari quanto lo stesso Francesco I e successori, persino Luigi XIV.

Con questo non si vuole - chiaramente - intendere o dire che re e parlamenti, ossia re e cortes, dovessero, o debbano, esser considerati come poteri paralleli ed eguali, di pari valore, autorità, prestigio, né qui, nei territori della Corona aragonese, né altrove, ossia che tutto procedesse tranquillamente in piena osservanza delle leggi, costituzioni e via dicendo. A giudicare dagli Atti, per esempio, delle Corts catalane, non vi era mossa del sovrano o dei funzionari da lui delegati a rappresentarlo, che non suscitasse rispettose ma ferme proteste e non venisse impugnata di illegittimità. In assenza, per esempio, nel 1420 di Alfonso V dai suoi Stati di terraferma, nelle Corts indette e presiedute, per suo mandato, dalla Regina, i Bracci s'inalberavano, una volta dopo l'altra, di tutto ciō ch'ella facesse o dichiarasse e ne chiedevano la correzione e la revoca. Ma non era tutto così semplice. A un certo punto, un certo giorno, la Regina (o chi per lei) faceva piazza pulita dei membri più eminenti del braccio 'militare', i conti di Pallars e di Cardona e vari altri Signori, impedendo loro, con l'arrestarli, di partecipare alle sedute: nei giorni precedenti, invero, ciascuno dei sunnominati conti aveva capeggiato, in contrasto l'uno con l'altro, una fazione o 'aderenza' del braccio militare, disputandosi la qualifica di maior part del dit stament. Nella seduta del 30 ottobre, il visconte d'Isola e Canneto si doleva del fatto, che ignorava le tradizionali benemerenze del Braccio, con la Regina, lamentando che ella volesse privarlo della sua libertà 'prendendo ed arrestando alcuni dei maggiori magnati, baroni e cavalieri, cosa mai vista in Cort, giacché la libertà consiste proprio nel poter parlare 
liberamente in propria difesa'. La Regina lì per lì non rispose, ed il braccio militare ne proponeva greuge, fatto, diceva, inaudito. Non fu, d'altronde, una questione di pronta soluzione e non risulta quando gli arrestati venissero messi in libertà. La conclusione formale dell' incidente non doveva aversi che il 22 febbraio 1421 , quando, in seduta, la Regina faceva leggere una sua dichiarazione, dicente che, visto il gravame presentato dai magnati, baroni e cavalieri del Principato contro l'arresto e imprigionamento dei conti di Pallars e di Cardona, del visconte di Evoll e di altri nobili e cavalieri facenti parte del bracio militare (erano tutti presenti alla seduta in cui tale dichiarazione veniva letta), operati 'ad istanza di parte' (non si precisava quale: forse gli stessi avvocati fiscali!), cosa, secondo il ricorso da essi presentato, contraria ai privilegi del re Pietro, che li proteggeva da simili arresti, vietando ogni procedimento di tal genere: essa pronunciava e dichiarava la nullità (!) di tali atti, da ritenere come non avvenuti (!) e reintegrava sia i singoli come il loro Braccio, e d'altro canto, lo stesso sovrano, nei loro diritti e pretogative rispettivi.

Si era trattato soltanto di una...svista? Non c'è da esserne troppo sicuri. La stessa regina Maria, a Saragozza, aveva fatto arrestare, afferrandolo personalmente, l'autorevolissimo Justicia del regno d'Aragona, Martino Diaz, malgrado tutte le sue immunità: il caso ci è stato - con un certo scandalo da parte sua, per tale enormità dal Belluga, con lusso di particolari. Si era trattato, soltanto, di una o due eccezioni alle prerogative delle Cortes, eccezioni che dovevano, secondo il proverbio, confermare la regola del rispetto di esse e dei membri di esse? Se mai, tali eccezioni sarebbero state non due, ma tre. Lo stesso Belluga, infatti, quale giudice dei gravami alle Corts valenzane, doveva esperimentare quanto poco valessero le immunità parlamentari, in quanto il fratello minore di Alfonso, fururo Giovanni II, lo avrebbe, per punirlo del suo zelo in tale qualitã, fatto imprigionare e chiudere, in compedibus ferreis, in una delle torri della città di Valenza e poi (dopo qualche mese e, probabilmente, per intervento dello stesso Alfonso) liberato, ma mandato in esilio, in Castiglia.

Tra crisi e depressioni economiche ed agitazioni sociali, i decen. ni di mezzo del secolo XV non furono, per la Catalogna, malgrado temporanei cedimenti dell' autorità regia, un periodo ideale neppure per le libertà e gli sviluppi parlamentari. Non solo in es$\mathrm{sa}$, comunque, $\mathrm{ma}$ in generale le istituzioni rappresentative 
-espressione, come pure superamento, della struttura feudale della società, integrata dal privilegio ecclesiastico e dall'autonomia cittadina mancarono e del prestigio e della capacità occorrenti per farne forze vive e direttive delle rispettive nazioni. Ê celebre -pure se alquanto tarda, postmedievale - l'affermazione, assai pessimistica, del giurista anglo-tolosano Guglielmo Barclay, secondo il quale: $1^{\circ}$ ) l'autorità delle assemblee, è somma soltanto nei periodi d'interregno, ma non di fronte ad un legittimo sovrano; $2^{\circ}$ ) gli 'stati', o parlamenti, senza il re non sono nulla, giacché egli può, anche senza il loro voto, stabilire tutto ciò ch'egli ritiene confacente al bene dello Stato; $3^{\circ}$ ) la Curia, l'assemblea, ha sempre bisogno del consenso del sovrano, mentre questi non ha affatto bisogno di quello dell'assemblea. La seconda di tali affermazioni sarebbe stata, come è noto, fatta propria e ripetuta, a metà circa del Settecento, dal celebre abate Mably nel suo De l'étude de l'bistoire: le roi peut beaucoup de choses sans le parlement; le parlement, au contraire, ne peut rien sans le roi. Nell'insieme, forse, Barclay aveva ragione. Ma la sua presa di posizione (fondatissima se riferita alla situazione francese e alla dappochezza de 'stati generali' del tempo) aveva il difetto, oltre che di essere troppo generica, di ignorare quel principio di contrattualismo al quale si è, qui, più volte accennato. Essa metteva troppo in ombra la realtà ed i frequenti accordi e compromessi tra i sovrani e le assemblee. Lapidario e deciso, uno scrittore ed operatore politico degli ultimi decenni del Quattrocento, Philippe de Commines aveva detto, e, forse, ammonito che il n'est en la puissance du prince au monde de lever impost a son plaistr sur le peuple, non plus que prendre le bien d'autruy. 